\title{
Kisspeptin regulates the development of caprine primordial follicles in vitro
}

\author{
Manjula Priyantha Sumith Magamage ${ }^{1,2}$, Sriravali Sathagopam ${ }^{1}$, Kiran Avula ${ }^{1}$, Di Neththi Nimesh \\ Madushanka $^{2}$ and Sathya Velmurugan ${ }^{1, *}$ \\ ${ }^{1}$ National Institute of Animal Biotechnology, Hyderabad 500049, India \\ ${ }^{2}$ Department of Livestock Production, Sabaragamuwa University, Belihuloya RN 70190, Sri Lanka
}

Received February 2, 2021

Revised March 15, 2021

Accepted March 18, 2021

\section{*Correspondence}

Sathya Velmurugan

E-mail: sathyavet@gmail.com

ORCID

https://orcid.org/0000-0003-0769-8424
ABSTRACT Kisspeptin, a neuropeptide and the master controller of reproductive axis upstream to $\mathrm{GnRH}$ neurons, and its receptor are also expressed in extrahypothalamic tissues, such as ovaries. As systemic kisspeptin has been shown to modulate follicular dynamics in cattle, we hypothesized that kisspeptin has direct actions on the ovarian follicular development. We also hypothesized that kisspeptin regulation of primordial follicle development is via modulation of VEGF expression. In order to test these hypotheses, we cultured caprine ovarian cortical strips in vitro for 7 days with supplementation of kisspeptin at 1, 10 and $100 \mu \mathrm{M}$ concentration and observed the development of primordial follicles into intermediate, primary and secondary follicles. We also studied the alteration in the expression profile of VEGF and VEGF transcript variant 2 mRNA during follicular development in the presence of kisspeptin. We confirmed the presence of GPR54 in goat ovaries in our preliminary studies. Supplementation of kisspeptin at 1 and $10 \mu \mathrm{M}$ concentration facilitated the development of primordial follicles into intermediate, primary and secondary follicles with less number of degenerated follicles while the same at $100 \mu \mathrm{M}$ resulted in degeneration of follicles. We observed a drastic increase in the expression profile of VEGF and VEGF transcript variant 2 mRNA upon culture which was independent of kisspeptin treatment. In conclusion, our studies show that kisspeptin facilitates ovarian primordial development in vitro.

Keywords: GPR54, kisspeptin, ovary, primordial follicle development, VEGF

\section{INTRODUCTION}

Kisspeptin, the product of the Kiss-1 gene, encodes a 145 -amino acid peptide that is further processed to generate biologically active peptides of various lengths (10-54 amino acids). Kisspeptin was initially described to have a central role in triggering the onset of puberty, however, it has been found to be involved in all phases of reproductive life (Caraty and Franceschini, 2008). Central or peripheral administration of kisspeptin strongly induces the secretion of gonadotropins mainly via stimulation of GnRH secretion (Caraty and Franceschini, 2008). Recently, kisspeptin has been shown to be able to synchronize preovulatory surges in cyclic ewes and cause ovulation in seasonally acyclic ewes (Gottsch et al., 2004). Further, kisspeptin and its receptor are expressed in extra-hypothalamic tissues such as ovaries (Uenoyama et al., 2016). Therefore, it is fundamentally important to study the role 
of kisspeptin during ovarian follicle differentiation in mammalian models to understand the major molecular mechanisms behind primordial follicle activation and follicle recruitment.

Stringent control of repeated cycles of growth and remodeling process is required in the ovary, as oocyte must develop in an avascular environment within the follicle, in contrast to the highly vascular corpus luteum (Zimmermann et al., 2001; Reynolds et al., 2002). Hence, the mammalian ovary undergoes programmed angiogenic processes during the ovarian cycle (Stouffer et al., 2001; Reynolds et al., 2002). Only the fastest growing and most highly aggressive tumors match the high rate of vessel growth and proliferation within the ovary which is associated with a high metabolic requirement for rapid steroidogenesis (Reynolds et al., 2002). Although the process of primordial follicle recruitment is incompletely understood, specific growth factors must either stimulate primordial follicles to leave the dormant state or inhibit primordial follicles from entering the growing pool (Kim, 2012). Among many candidates, vascular endothelial growth factor-A (VEGF-A), a member of the VEGF family, has emerged as one of the most important regulator of angiogenesis in the ovary (Geva and Jaffe, 2000). The $V E G F-A$ gene consists of eight exons, which undergo alternative splicing to form different mRNA splice variants and are translated into VEGF-A protein isoforms with different numbers of amino acids (Arcondéguy et al., 2013). VEGF is essential to vasculogenesis (Drake et al., 2000). Through alternative mRNA splicing, the VEGF-A isoforms differ by the presence or absence of sequences encoded by exons 6 and 7 (Tischer et al., 1991). VEGF-A has been known to promote proliferation and migration of vascular endothelial cells, and to enhance vascular permeability (Senger et al., 1983, 1993; Leung et al., 1989; Pepper et al., 1992). Its expression in the ovary undergoes dynamic changes during follicle maturation, ovulation and luteinization (Stouffer et al., 2001). VEGF mRNA is upregulated during the primordial to primary follicle transition in postnatal rat ovaries (Kezele et al., 2005). In vivo injections of VEGF antibody hinders the development of primordial follicles (Roberts et al., 2007). These findings are noteworthy as there are no blood vessels around the primordial or primary follicles (McFee et al., 2009).

There are limited number of studies on the role of VEGF in ovarian physiology in domestic animal species. Here we explored the expression of VEGF-A transcript variant 2 and VEGF-A165a, proangiogenic factors, during follicle development in goat ovaries in vitro. Kisspeptin, as a metastasis inhibitor, inhibits angiogenesis (Cho et al., 2009). Hence, we hypothesized that kisspeptin reduces VEGF$A$ expression during ovarian follicular development. The objective of the study was to explore VEGF-A expression in caprine cortical strips that are treated with kisspeptin.

We found that kisspeptin did not suppress the increase in VEGF expression observed during the culture of ovarian cortical strips. Further, kisspeptin facilitated primordial follicle development in line with the in vivo observation in livestock on ovarian follicular dynamics. We believe that understanding the role of kisspeptin and VEGF cascades during follicle development may provide a new set of tools to address infertility of ovarian origin and assisted reproduction. It may also have an impact on the control of metastatic ovarian tumors.

\section{MATERIALS AND METHODS}

\section{Collection of ovarian cortical strips}

Goat ovaries, along with uteri, were collected from a slaughter house and transported to the laboratory under controlled conditions. Ovaries were collected, cleaned, washed once in phosphate buffered saline (PBS) supplemented with $0.2 \%(\mathrm{w} / \mathrm{v})$ cetyltrimethylammonium bromide and washed thrice in Dulbecco's PBS (Sigma) supplemented with $0.1 \%(\mathrm{w} / \mathrm{v})$ polyvinyl alcohol. Ovarian cortical strips (approximately, $2 \mathrm{~mm} \times 1 \mathrm{~mm} \times 0.5 \mathrm{~mm}$ ) that contain primordial follicles were selected, and cut into two pieces (each approximately $1 \mathrm{~mm} \times 1 \mathrm{~mm} \times 0.5 \mathrm{~mm}$ ); one part was fixed immediately for histological examination later to assess the follicle number and morphology and the other part was washed thrice and immersed in Leibovitz 15 medium (Sigma) before culture. The Leibovitz medium contained $0.1 \%$ (v/v) Pen-Strep as antibiotic.

\section{In vitro culture}

In each experiment, a group of 8 cortical strips were cultured for $168 \mathrm{~h}$ on a floating membrane filter (diameter $25 \mathrm{~mm}$ and pore size $0.45 \mu \mathrm{m}$ ) in six-well culture dish with Millicell ${ }^{\circledR}$ Cell Culture Inserts (Millipore) under the humidified atmosphere of $5 \% \mathrm{CO}_{2}$ and $95 \%$ air at $38^{\circ} \mathrm{C}$. The basic culture medium is Dulbecco's Minimum Essential Medium (D-MEM; Sigma) supplemented with Pen- 
Strep (1\% v/v), sodium pyruvate $(0.1 \mathrm{mg} / \mathrm{mL})$, InsulinTransferrin-Selenium (ITS; 0.05\% v/v) and bovine serum albumin $(5 \% \mathrm{v} / \mathrm{v})$. During the culture period, half of the total medium was changed every $24 \mathrm{~h}$. Either saline or kisspeptin-10 (1, 10 or $100 \mathrm{ng} / \mathrm{mL}$; equivalent to 0.75 , 7.59 and $75.85 \mathrm{nM}$, respectively; Tyr-Asn-Trp-Asn-SerPhe-Gly-Leu-Arg-Tyr-NH2; synthesized from Biotech Desk) was added on day 1.

\section{Histology and assessment of follicle development}

The ovarian cortical strips before and after culture were fixed in $4 \%(\mathrm{w} / \mathrm{v})$ paraformaldehyde in PBS, dehydrated, embedded in paraffin wax and serially sectioned (10 $\mu \mathrm{m}$ sections), and stained with hematoxylin and eosin. Primordial follicles were identified as those having oocytes of $25-30 \mu \mathrm{m}$ diameter containing a large spherical nucleus surrounded by small lipid droplets (Magamage et al., 2010). The numbers of different stages of follicles were recorded. The follicles were counted in every section where the oocyte nucleus was seen and hence double counting in adjacent sections was avoided. The follicles were classified into four categories according to the number and morphology of granulosa cell layers: (1) primordial follicles with a single layer of flattened granulosa cells surrounding the oocyte, (2) intermediate follicles with a single layer containing a mixture of flat and cuboidal granulosa cells, (3) primary follicles with a single layer of cuboidal granulosa cells, and (4) secondary follicles with two or more layers of cuboidal granulosa cells (Magamage et al., 2010). Degenerated follicles were identified by the staining properties of the oocyte cytoplasm and nucleus: pale cytoplasm and dark pyknotic nucleus. Furthermore, follicles having a shrunken oocyte, extensive cytoplasmic vacuolations and disintegrated granulosa cell layer were also considered to be degenerated follicles.

\section{Western blot}

The ovarian cortical strips were washed thrice in PBS before and after culture; protein extraction was done using RIPA buffer and quantification using Bradford assay. Rabbit polyclonal anti-GPR54 antibody (GeneTex; Cat No. GTX37417) was used for probing. HRP-conjugated goat anti-rabbit IgG antibody was used as the secondary antibody. A Western blotting detection kit (ECL Prime; GE; Cat. No. RPN2232) was used for detection.

\section{Quantitative PCR for Kiss1, GPR54, VEGF and VEGF Trans Var2 mRNA}

The ovarian cortical strips were washed thrice in PBS before and after culture and RNA extraction was done using RNEasy plus mini kit (Qiagen) following the manufacturer's protocol and quantified by Nano Drop 2000 (Thermo Scientific). cDNA was synthesized using a kit (Takara; Cat \# 6110A) by reverse transcription using 200 ng of RNA. The sequences of the primer sets (IDT) are given in Table 1 . The assays were performed in a Real Time PCR System (Applied Biosystems 7500 ). $\beta$-actin was used to normalize the input of $\mathrm{CDNA}$ in $\mathrm{qPCR}$. The ratio of target gene compared with $\beta$-actin amplification represented the relative levels in each sample. Results were calculated based on $\mathrm{Ct}$ values using the formula $2 \wedge-[\mathrm{Ct}$ (gene)-Ct ( $\beta$-actin)] as described in the user manual (Applied Biosystems).

\section{Statistics}

Data were compiled using Excel (Microsoft Inc). GraphPad Prism (Version 6.07, GraphPad Software Inc.) statistical software was used to analyse the data using one-way ANOVA followed by Tukey's multiple comparison tests. Differences were considered to be significant when $p<$ 0.05 .

\section{RESULTS}

\section{GPR54 expression in caprine ovary}

In preliminary studies, the presence of kisspeptin recep-

Table 1. Primers used for qPCR

\begin{tabular}{ll}
\hline VEGF-A165a & \\
Forward primer & GTTCAGAGCGGAGAAAGCAT \\
Reverse primer & TCACATCTGCAAGTACGTTCG \\
Capra hircus VEGF-A transcript variant 2 \\
$\begin{array}{l}\text { Forward primer } \\
\text { Reverse primer }\end{array}$ & AACCTGACATGAAGGAAGAGGGAG \\
Capra hircus Kisspeptin 1 & \\
Forward primer & TCCCTCCCTTCTTTCCTTCCTAA \\
Reverse primer & AGGGACGAGCCTGAACCGA \\
Capra hircus GPR54 & \\
Forward primer & GTCTGGGAAGAAGGTTGGGAG \\
Reverse primer & CCGTCTTGGGTTTCCATTGTG \\
Capra hircus $\beta$-actin & \\
Forward primer & GTCACCAACTGGGACGACAT \\
Reverse primer & CATCTTCTCACGGTTGGCT \\
\hline
\end{tabular}


tor, GPR54, in ovarian cortical strips, was confirmed by Western blot analysis (Fig. 1).

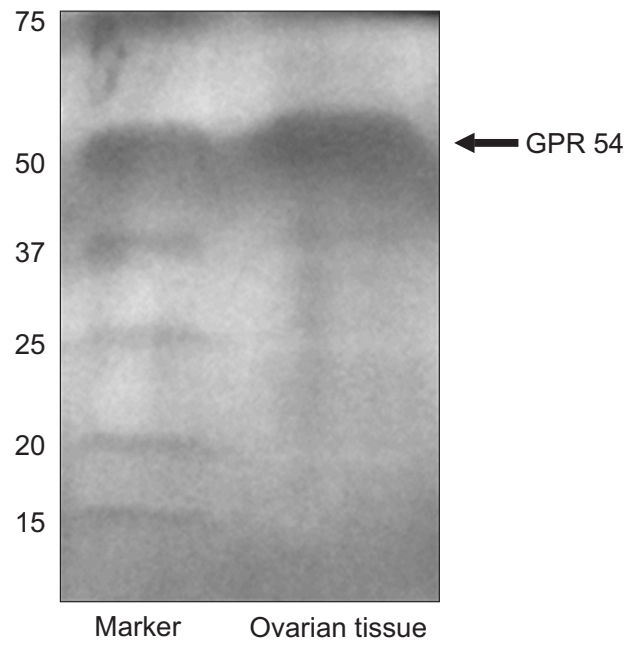

Fig. 1. Expression of GPR54, the kisspeptin receptor, in pre-pubertal goat ovarian cortical strips.

\section{Effect of kisspeptin on primordial follicle development}

Kisspeptin treatment induced development of primordial follicles into primary and secondary follicles upon culture. Representative histological sections depicting various stages of follicle development with respect to treatments are shown in Fig. 2.

On day 0 , prior to culture, the cortical strips predominantly contained primordial follicles. Upon culture without any treatment, there was no development of these follicles. Upon supplementation of kisspeptin, primordial follicles developed into intermediate, primary and secondary follicles. While kisspeptin at $1 \mathrm{ng} / \mathrm{mL}$ concentration facilitated the development of primordial follicles into intermediate, primary and secondary follicles with least number of degenerated follicles, $10 \mathrm{ng} / \mathrm{mL}$ kisspeptin facilitated the same with lesser number of degenerated follicles (Fig. 3A to 3E). Degenerated follicles were the highest with kisspeptin at $100 \mathrm{ng} / \mathrm{mL}$ concentration (Fig. 3E). Total number of viable follicles were the highest in $1 \mathrm{ng} / \mathrm{mL}$ kisspeptin-treated strips and the least in 100
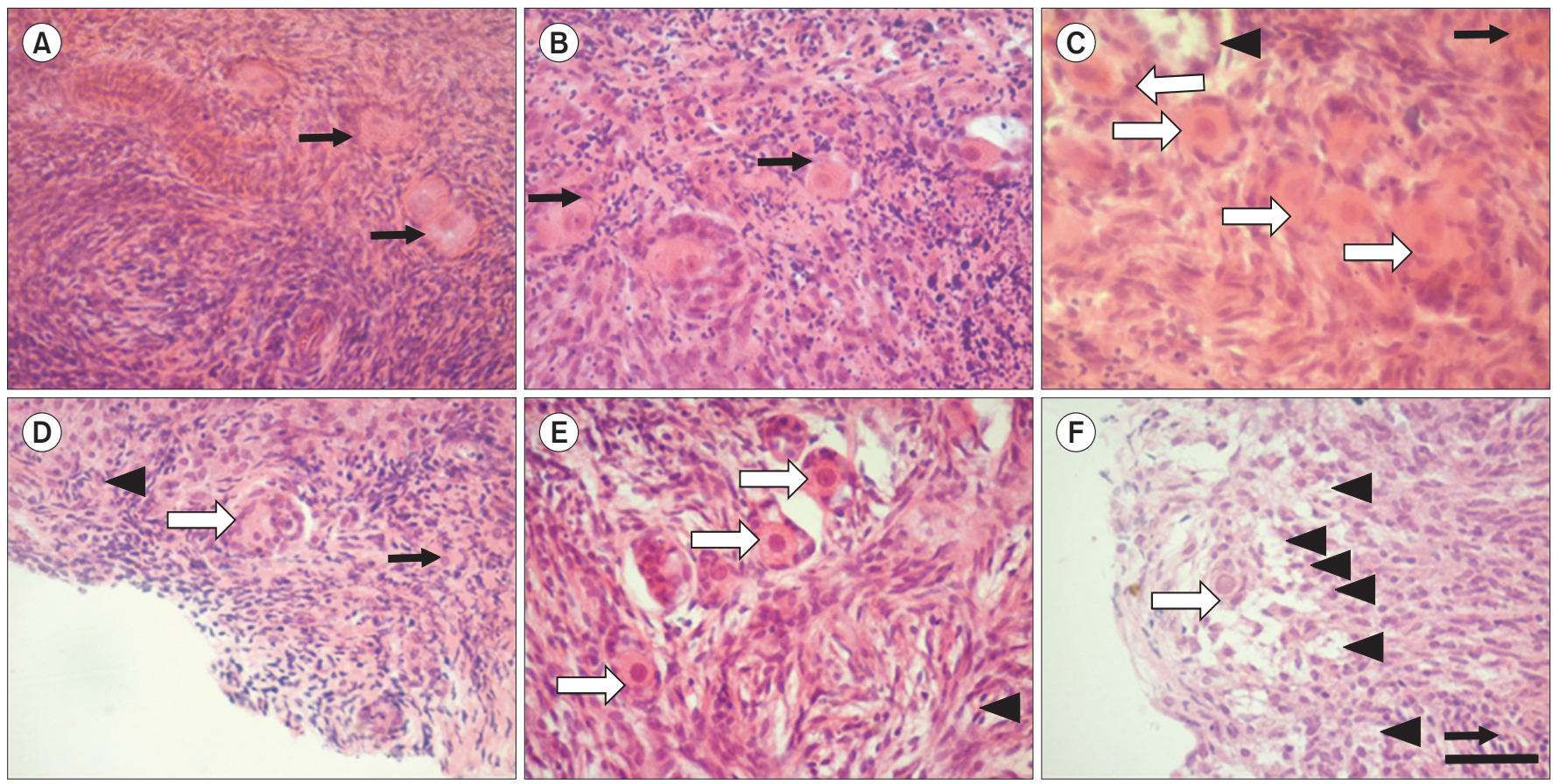

Fig. 2. Representative histological sections, stained with Haematoxylin and Eosin, from caprine ovarian cortical strips cultured in vitro for 7 days with three different concentrations of kisspeptin. (A) Day 0 - uncultured control; (B) Day 7 - cultured control; (C) and (D) Day 7 with kisspeptin at $1 \mathrm{ng} / \mathrm{mL}$; (E) Day 7 - with kisspeptin at $10 \mathrm{ng} / \mathrm{mL}$; (F) Day 7 - with kisspeptin at $100 \mathrm{ng} / \mathrm{mL}$. Magnification: 40×; scale bar: $100 \mu \mathrm{m}$. Follicle viability as well as number of developing follicles were prominent in sections from $1 \mathrm{ng} / \mathrm{mL}$ and $10 \mathrm{ng} / \mathrm{mL}$ kisspeptin-supplemented strips while wide spread follicle degeneration was evident with $100 \mu \mathrm{M}$ kisspeptin treatment. While primordial follicles (black arrow), with flat granulosa cells indistinct from ovarian stroma, are prominent in control strips, primary follicles (white arrow), with cuboidal granulosa cells visible around the oocyte, are more in $1 \mathrm{ng} / \mathrm{mL}$ and $10 \mathrm{ng} / \mathrm{mL}$ kisspeptin-treated strips. Degenerated follicles (black arrow heads) were more in $100 \mathrm{ng} / \mathrm{mL}$ kisspeptin-treated strips. 
ng/mL kisspeptin-treated strips (Fig. 3F).

\section{Kisspeptin and VEGF expression profile during primordial follicle development}

There was no significant change in the expression of kiss1 and GPR54 mRNA in the ovarian cortical strips during in vitro culture (Fig. $4 \mathrm{~A}$ and $4 \mathrm{~B}$ ). On the contrary, the mRNA expression of VEGF- $A_{165}$ a and VEGF-A transcript variant 2 drastically increased upon culture in comparison to day 0 (Fig. 4C and 4D). This increase in expression was irrespective of kisspeptin treatment.

\section{DISCUSSION}

Kisspeptin, acting via its receptor, is a critical regula- tor of the reproductive axis by stimulating hypothalamic GnRH release (Caraty and Franceschini, 2008). More recently, studies have suggested that kisspeptin may also have direct gonadal effects due to the presence of GPR54 in ovaries (Castellano et al., 2006). The current study on the role of kisspeptin on VEGF expression and on primordial follicle development in mammalian ovaries delineates yet another significant role played by kisspeptin in ovaries.

The majority of the follicles in the ovary are the primordial follicles (Findlay et al., 2015). Though sporadic development of these follicles into other developmental stages are observed even before puberty, development of these follicles into advanced stages, such as tertiary and ovulatory follicles, occur upon the influence of reproduc-
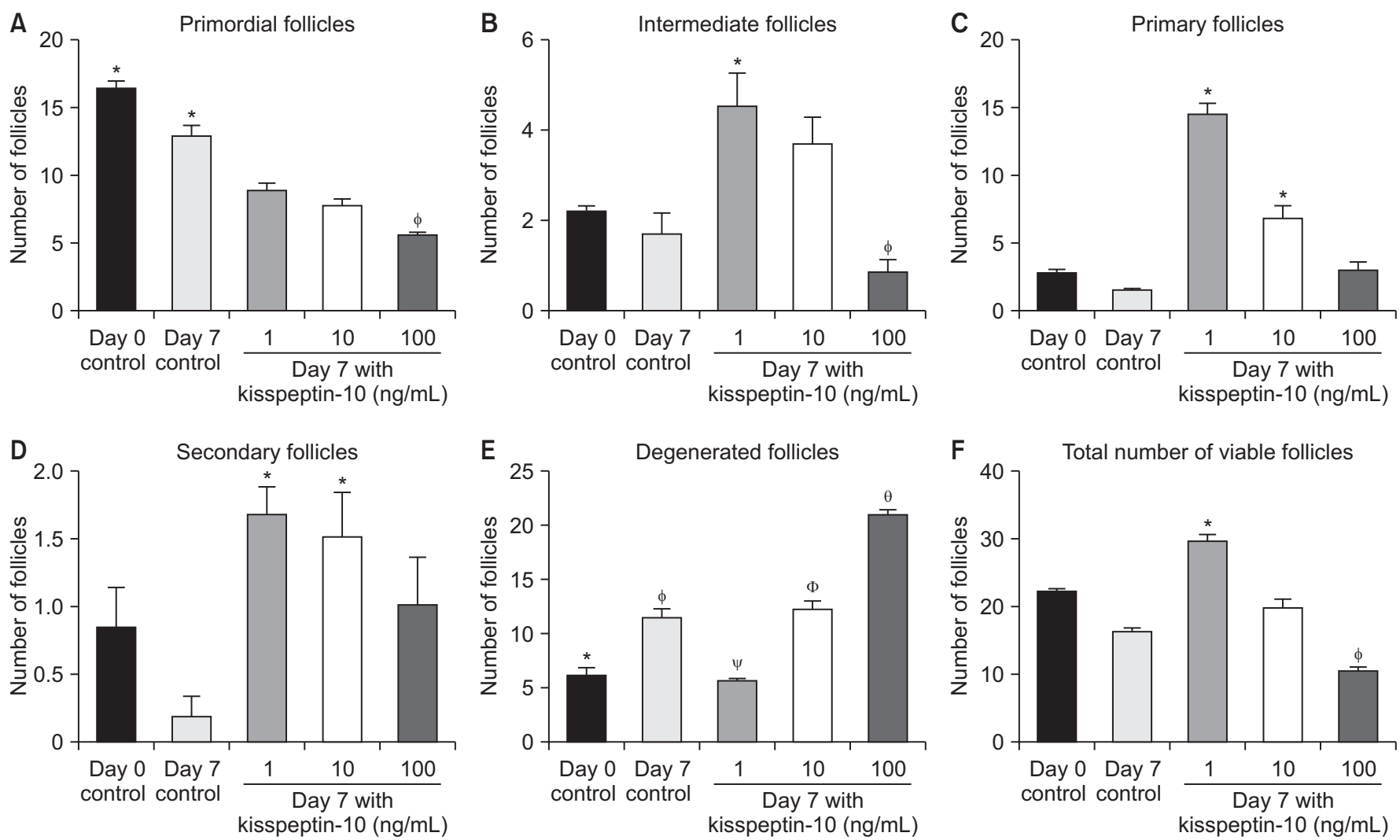

Fig. 3. Effect of kisspeptin on caprine primordial follicle development in vitro. (A) Primordial follicles, (B) Intermediate follicles, (C) Primary follicles, (D) Secondary follicles, (E) Degenerated follicles, and (F) Total number of viable follicles. During culture of ovarian cortical strips for 7 days, supplementation of kisspeptin at $1 \mathrm{ng} / \mathrm{mL}$ concentration facilitated development of primordial follicles into intermediate, primary and secondary follicles with least number of degenerated follicles. Supplementation of kisspeptin at $10 \mathrm{ng} / \mathrm{mL}$ concentration also facilitated development of primordial follicles into primary and secondary follicles with lesser number of degenerated follicles while kisspeptin at $100 \mathrm{ng} / \mathrm{mL}$ resulted in degeneration of follicles (statistics: primordial follicles: *vs. all other groups; $\phi$ vs. day 7 Kiss-10 $1 \mu \mathrm{M}$ and $10 \mu \mathrm{M}$; intermediate follicles: *vs. control groups; $\phi$ vs. day 7 Kiss-10 $1 \mu \mathrm{M}$ and $10 \mu \mathrm{M}$; primary follicles: *vs. all other groups; secondary follicles: ${ }^{*}$ vs. control groups; degenerated follicles: *vs. other groups except day 7 Kiss-10 $1 \mu \mathrm{M} ; \phi$ vs. other groups except day 7 Kiss-10 $10 \mu \mathrm{M}$; $\Psi$ vs. other groups except day 0 control; $\Phi$ vs. other groups except day 7 control; $\theta$ vs. other groups; total number of viable follicles: ${ }^{*} v s$. other groups; $\phi$ vs. other groups; $p<0.05$, one-way ANOVA followed by Tukey's multiple comparison test). Date represented as mean \pm SEM; $n=6-8$ separate experiments. 

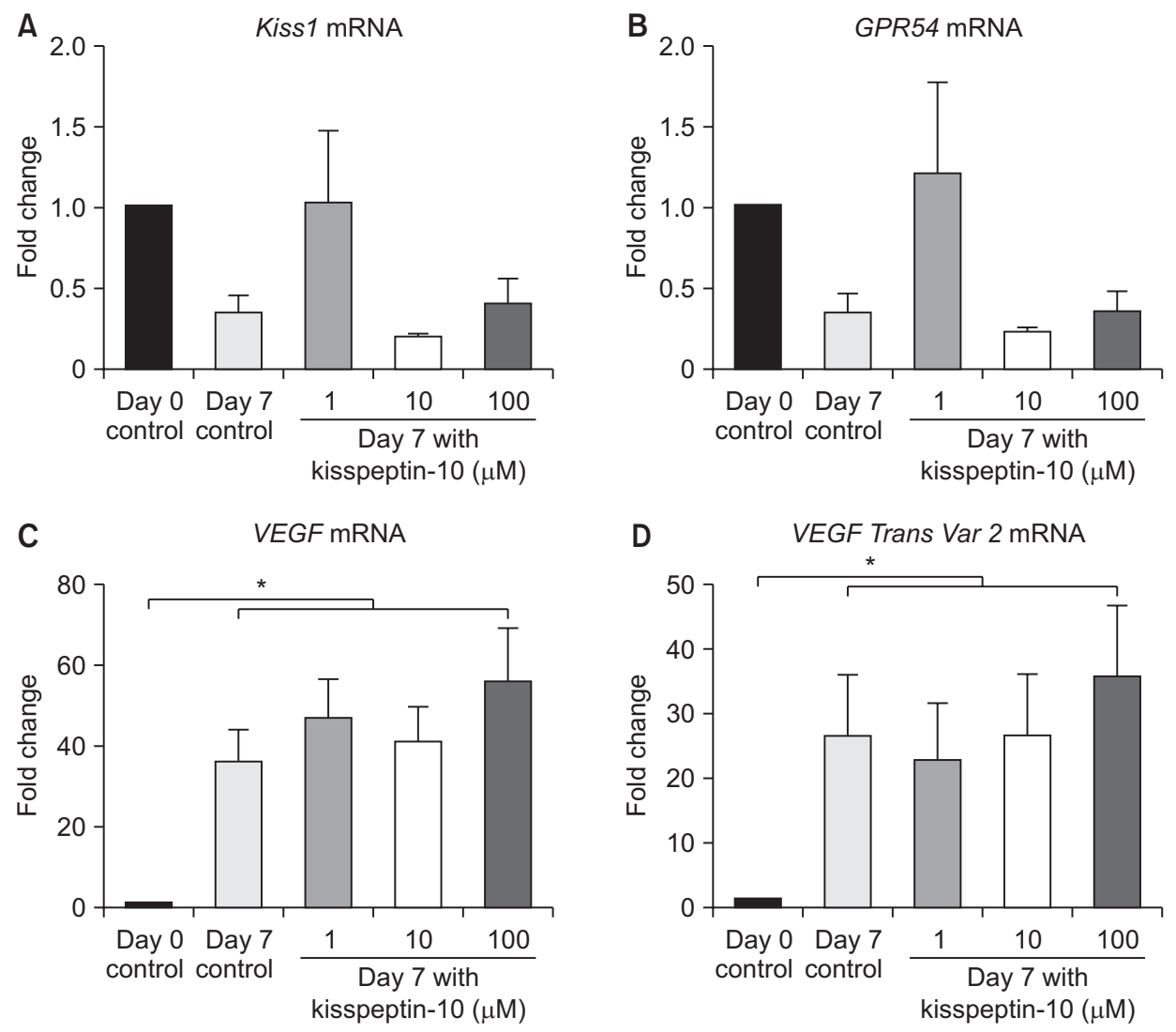

Fig. 4. Expression profiles of Kiss1, GPR54, VEGF and VEGF Transcript variant 2 mRNA in cortical strips upon in vitro culture. The drastic increase in the expression profile of VEGF and VEGF Transcript variant 2 mRNA upon culture ( ${ }^{*} v s$. Day $0-$ Uncultured control, $p<0.0001$, one-way ANOVA) is independent of kisspeptin-10 treatment.

tive hormones that are released during regular oestrous or menstrual cycles. Facilitation of development of primordial follicles into other subsequent stages, by kisspeptin, as observed in our study, demonstrates that hypothalamic or ovarian kisspeptin plays an important role in the regulation of ovarian physiology, as reviewed recently (Clarke et al., 2015).

Angiogenesis is an important event during primordial follicle development as the blood supply increases to support the growing follicle (Fraser, 2006). Hence, increase in the expression of VEGF during follicle development may be expected. However, increase in the same in the 7-day cultured control strips, in which there is no significant follicle development was observed, is intriguing.

Kisspeptin, while inhibiting angiogenesis during metastasis, did not inhibit VEGF, an angiogenic factor, in the ovarian strips, as observed in our study. Though most of the studies have emphasized the anti-angiogenic effect of kisspeptin, especially during tumour growth, a few studies have shown that kisspeptin increases migration and proliferation of endothelial cells, the components of angiogenesis (Golzar and Javanmard, 2015). Facilitation by kisspeptin of ovarian follicular growth has been observed in vivo in livestock species (Pottapenjera et al, 2018). The current study supports the in vivo observation and further suggests a direct action of kisspeptin on ovaries rather than indirect effects via release of gonadotropins.

In conclusion, this study demonstrates a direct action of kisspeptin on ovarian cells facilitating primordial follicle development. In addition, lack of inhibition of VEGF expression by kisspeptin suggests proangiogenic effects of kisspeptin in the ovary. The results from this in vitro study may be evaluated in an in vivo model for further corroboration. This study paves way for further exploration on whether kisspeptin would be an ideal therapeutic molecule for treating ovarian disorders leading to infertility in humans as well as animals. 


\section{CONFLICTS OF INTEREST}

The study was conducted at NIAB. Authors are not affiliated with NIAB at the time of publication.

\section{FUNDING}

Funding was partially supported by India Science and Research Fellowship to MPSM by the Department and Ministry of Science and Technology, Government of India. KA received Junior Research Fellowship from a project (No. PR12395) funded by Department of Biotechnology, Government of India.

\section{ACKNOWLEDGEMENTS}

Research infrastructure and manpower support by NIAB to SV is acknowledged.

\section{AUTHOR CONTRIBUTIONS}

MPSM conceived the idea, designed and performed experiments, compiled and analyzed the data; SS assisted in culture experiments, performed qPCR and Western blot, and assisted in microtome sectioning; KA collected samples, and assisted in culture experiments; DNNM assisted in data analysis; SV compiled and analyzed the data, prepared the figures and wrote the manuscript.

\section{AUTHOR'S POSITION AND ORCID NO.}

\author{
MPS Magamage, Professor, \\ https://orcid.org/0000-0003-1227-3607 \\ $S$ Sathagopam, Project Fellow, \\ https://orcid.org/0000-0003-2249-0883 \\ K Avula, PhD Student, \\ https://orcid.org/0000-0003-0014-1009 \\ DNN Madushanka, Demonstrator, \\ https://orcid.org/0000-0003-1704-7123 \\ S Velmurugan, Post-doctoral Research Scholar, \\ https://orcid.org/0000-0003-0769-8424
}

\section{REFERENCES}

Arcondéguy T, Lacazette E, Millevoi S, Prats H, Touriol C. 2013. VEGF-A mRNA processing, stability and translation: a paradigm for intricate regulation of gene expression at the post- transcriptional level. Nucleic Acids Res. 41:7997-8010.

Caraty A and Franceschini I. 2008. Basic aspects of the control of GnRH and LH secretions by kisspeptin: potential applications for better control of fertility in females. Reprod. Domest. Anim. 43 Suppl 2:172-178.

Castellano JM, Gaytan M, Roa J, Vigo E, Navarro VM, Bellido C, Dieguez C, Aguilar E, Sánchez-Criado JE, Pellicer A, Pinilla L, Gaytan F, Tena-Sempere M. 2006. Expression of KiSS-1 in rat ovary: putative local regulator of ovulation? Endocrinology 147:4852-4862.

Cho SG, Yi Z, Pang X, Yi T, Wang Y, Luo J, Wu Z, Li D, Liu M. 2009. Kisspeptin-10, a KISS1-derived decapeptide, inhibits tumor angiogenesis by suppressing Sp1-mediated VEGF expression and FAK/Rho GTPase activation. Cancer Res. 69:7062-7070.

Clarke H, Dhillo WS, Jayasena CN. 2015. Comprehensive review on kisspeptin and its role in reproductive disorders. Endocrinol. Metab. (Seoul) 30:124-141.

Drake CJ, LaRue A, Ferrara N, Little CD. 2000. VEGF regulates cell behavior during vasculogenesis. Dev. Biol. 224:178-188.

Findlay JK, Hutt KJ, Hickey M, Anderson RA. 2015. How is the number of primordial follicles in the ovarian reserve established? Biol. Reprod. 93:111.

Fraser HM. 2006. Regulation of the ovarian follicular vasculature. Reprod. Biol. Endocrinol. 4:18.

Geva E and Jaffe RB. 2000. Role of vascular endothelial growth factor in ovarian physiology and pathology. Fertil. Steril. 74:429-438.

Golzar F and Javanmard SH. 2015. The effects of kisspeptin-10 on migration and proliferation of endothelial cell. Adv. Biomed. Res. 4:41.

Gottsch ML, Cunningham MJ, Smith JT, Popa SM, Acohido BV, Crowley WF, Seminara S, Clifton DK, Steiner RA. 2004. A role for kisspeptins in the regulation of gonadotropin secretion in the mouse. Endocrinology 145:4073-4077.

Kezele PR, Ague JM, Nilsson E, Skinner MK. 2005. Alterations in the ovarian transcriptome during primordial follicle assembly and development. Biol. Reprod. 72:241-255.

Kim JY. 2012. Control of ovarian primordial follicle activation. Clin. Exp. Reprod. Med. 39:10-14.

Leung DW, Cachianes G, Kuang WJ, Goeddel DV, Ferrara N. 1989. Vascular endothelial growth factor is a secreted angiogenic mitogen. Science 246:1306-1309.

Magamage MPS, Zengyo M, Moniruzzaman M, Miyano T. 2010. Testosterone induces activation of porcine primordial follicles in vitro. Reprod Med Biol. 10:21-30.

McFee RM, Artac RA, McFee RM, Clopton DT, Smith RA, Rozell TG, Cupp AS. 2009. Inhibition of vascular endothelial growth factor receptor signal transduction blocks follicle progression but does not necessarily disrupt vascular development in perinatal rat ovaries. Biol. Reprod. 81:966-977.

Pepper MS, Ferrara N, Orci L, Montesano R. 1992. Potent synergism between vascular endothelial growth factor and basic fibroblast growth factor in the induction of angiogenesis in vitro. Biochem. Biophys. Res. Commun. 189:824-831. 
Pottapenjera V, Rajanala SR, Reddy C, Gangineni A, Avula K, Bejjanki SK, Sathagopam S, Kesharwani S, Velmurugan S. 2018. Kisspeptin modulates luteinizing hormone release and ovarian follicular dynamics in pre-pubertal and adult Murrah buffaloes. Front. Vet. Sci. 5:149.

Reynolds LP, Grazul-Bilska AT, Redmer DA. 2002. Angiogenesis in the female reproductive organs: pathological implications. Int. J. Exp. Pathol. 83:151-163.

Roberts AE, Arbogast LK, Friedman CI, Cohn DE, Kaumaya PT, Danforth DR. 2007. Neutralization of endogenous vascular endothelial growth factor depletes primordial follicles in the mouse ovary. Biol. Reprod. 76:218-223.

Senger DR, Galli SJ, Dvorak AM, Perruzzi CA, Harvey VS, Dvorak HF. 1983. Tumor cells secrete a vascular permeability factor that promotes accumulation of ascites fluid. Science 219:983-985.

Senger DR, Van de Water L, Brown LF, Nagy JA, Yeo KT, Yeo TK, Berse B, Jackman RW, Dvorak AM, Dvorak HF. 1993. Vascu- lar permeability factor (VPF, VEGF) in tumor biology. Cancer Metastasis Rev. 12:303-324.

Stouffer RL, Martínez-Chequer JC, Molskness TA, Xu F, Hazzard TM. 2001. Regulation and action of angiogenic factors in the primate ovary. Arch. Med. Res. 32:567-575.

Tischer E, Mitchell R, Hartman T, Silva M, Gospodarowicz D, Fiddes JC, Abraham JA. 1991. The human gene for vascular endothelial growth factor. Multiple protein forms are encoded through alternative exon splicing. J. Biol. Chem. 266:11947-11954.

Uenoyama Y, Pheng V, Tsukamura H, Maeda KI. 2016. The roles of kisspeptin revisited: inside and outside the hypothalamus. J. Reprod. Dev. 62:537-545.

Zimmermann RC, Xiao E, Husami N, Sauer MV, Lobo R, Kitajewski J, Ferin M. 2001. Short-term administration of antivascular endothelial growth factor antibody in the late follicular phase delays follicular development in the rhesus monkey. J. Clin. Endocrinol. Metab. 86:768-772. 